\title{
INELASTIC MEAN FREE PATHS OF LOW-ENERGY ELECTRONS IN SOLIDS
}

\author{
S. TANuma \\ Analysis Research Center, Nippon Mining Company Ltd. \\ 3-17-35 Nizo-Minami, Toda, Saitama 335, Japan \\ C.J. Powell and D.R. PenN \\ National Institute of Standards and Teclinology, Gaithersburg, MD 20899, USA \\ (Received May 21, 1991)
}

We present a summary of recent calculations of the elcctron inelastic mean free paths (IMFPs) of 50-2000 eV electrons in 27 elcments and 15 inorganic compounds. These calculations are based in part on experimental optical data to represent the dependence of the inelastic scattering probability on energy loss and the theoretical Lindhard dielectric function to represent the dependence of the scattering probability on momentum transfer. The calculated IMFPs for the elements were fitted to a modified form of the Bethe equation for inelastic electron scattering in matter and the four parameters in this equation were empirically related to other material parameters. The resulting formula, designated TPP-2, provides a convenient means for predicting IMFPs in other materials. We have used two powerful integral equations or sum rules to evaluate the optical data on which our IMFP calculations are based. While the optical data for the elements satisfied these sum rules to an acceptable degree, there were significant deviations in the data for the compounds. In addition, differences in IMFPs calculated from the optical data for the compounds and the values predicted by TPP-2 correlated with the average errors of the optical data as determined by the sum rules. IMFPs calculated from TPP-2 for these compounds are therefore believed to be more reliable than IMFPs obtained from the imperfect optical data.

PACS numbers: $82.80 . P v, 79.20 . F v, 79.60 .-\mathrm{i}, 78.90 .+\mathrm{t}$

\section{Introduction}

The surface sensitivity of electron spectroscopies such as Auger electron spectroscopy (AES) and X-ray photoelectron spectroscopy (XPS) depends on the mean free paths for both inelastic and elastic scattering in the material of interest. Experimental efforts to determine surface sensitivity have generally involved measurements of a parameter termed the electron attenuation length $(\mathrm{AL})$ which is 
currently not well defined [1-4]. For those few materials for which multiple AL measurements have been made in different laboratories under similar conditions, there are discrepancies of up to about a factor of two between the lowest and highest reported $A L$ values at a given electron energy $[5,6]$. These discrepancies are believed to be mainly due to the many sources of systematic error in $A \mathrm{~L}$ measurements [1]. The likelihood of substantial systematic errors has made it difficult to evaluate the extent to which measured ALs depend on the experimental configuration, as has been predicted from transport calculations $[2-4,7,8]$. It has also been similarly difficult to determine with a high degree of confidence how ALs vary with chemical state or composition and with electron energy [1].

We present here some results of recent calculations of electron inelastic mean free paths (IMFPs) of 50-2000 eV electrons in 27 elemental solids and 15 inorganic compounds [6]. The calculations are based on an algorithm due to Penn [9] in which experimental optical data for each material are used to represent the dependence of the inelastic scattering probability on energy loss and the theoretical Lindhard dielectric function [10] is used to represent the dependence of the scattering probability on momentum transfer. Details of these calculations have been reported elsewhere [6]. The IMFP results for the group of 27 elements have been fitted to a modified form of the Bethe equation for inelastic electron scattering in matter [11]. It was found that the four parameters in this equation could be empirically related to several material parameters (atomic weight, density, and number of valence electrons per atom). The modified Bethe equation together with the relations for its parameters constitute an equation, to be referred to as TPP-2, for predicting IMFPs in other materials.

We have compared IMFP values calculated for the group of 15 inorganic compounds with those predicted by TPP-2 for each material [6]. For most of these compounds, the IMFPs calculated with the Penn algorithm were larger than the TPP-2 values. This systematic discrepancy was correlated with inadequacies of the optical data on which the IMFP calculation is based. We demonstrate here the value of two sum rules for evaluating sets of optical data of the type we have utilized.

\section{IMFP results}

The IMFP is calculated from a model diclectric function $\varepsilon(\omega, q)$ which is a function of frequency $\omega$ and momentum transfer $q[9,12]$. The cross-section for inelastic scattering involving energy loss $\hbar \omega$ and momentum transfer $q$ is proportional to $\operatorname{Im}[-1 / \varepsilon(\omega, q)]$. We make use of experimental optical data to compute $\operatorname{Im}[-1 / \varepsilon(\omega, 0)]$ for each material of interest. Since there is little experimental data a vailable concerning the $q$-dependence of $\varepsilon(\omega, q)$, we have used the theoretical expression of Lindhard et al. [10] to give a physically reasonable representation of $\varepsilon(\omega, q)$. The total inelastic scattering cross-section and thus the IMFP can then be found by a numerical integration over the kinematically allowed ranges of energy loss and momentum transfer.

IMFPs for the elements at energies between 50 and $200 \mathrm{eV}$ were calculated from Eq. (14) of Ref. [12] which involves a triple integration over $q, \omega$, and an 
electron density variable in the Lindhard expression. For energies above $200 \mathrm{eV}$, the IMFPs were calculated from Eq. (16) of Ref. [12] which requires only a single integration over $\omega$ [6]. For the compounds, Eq. (14) of Ref. [12] was used for energies between 50 and $800 \mathrm{eV}$ and Eq. (16) was used for energies up to $2000 \mathrm{eV}$. All energies are expressed with respect to the Fermi level.

The IMFPs were calculated for 27 elements and 15 inorganic compounds for which experimental optical data were available over most of the photon energy range 1-10,000 eV [6]. For over half of the materials, there were gaps in the data, often in the range 40-100 eV; in such cases, we made interpolations based on atomic photoabsorption calculations [13]. The consistency of the optical data was checked with two sum rules that are given below. Based on this analysis, the root-mean-square (RMS) uncertainty of the optical data for the elements was found to be about $10 \%$ while that for the compounds was larger, as will be discussed below. There are additional uncertainties in the calculated IMFPs associated with approximations in our algorithm [6]. These uncertainties are difficult to estimate reliably but are expected to be larger at lower energies $(50-200 \mathrm{eV})$ than at higher energies. Nevertheless, since we are using the same algorithm in a consistent way to calculate IMFPs in a large number of materials, we believe that we can determine the IMFP variations from material to material with higher accuracy than with individual IMFP values themselves. We can similarly determine the IMFP dependence on electron encrgy with high precision.
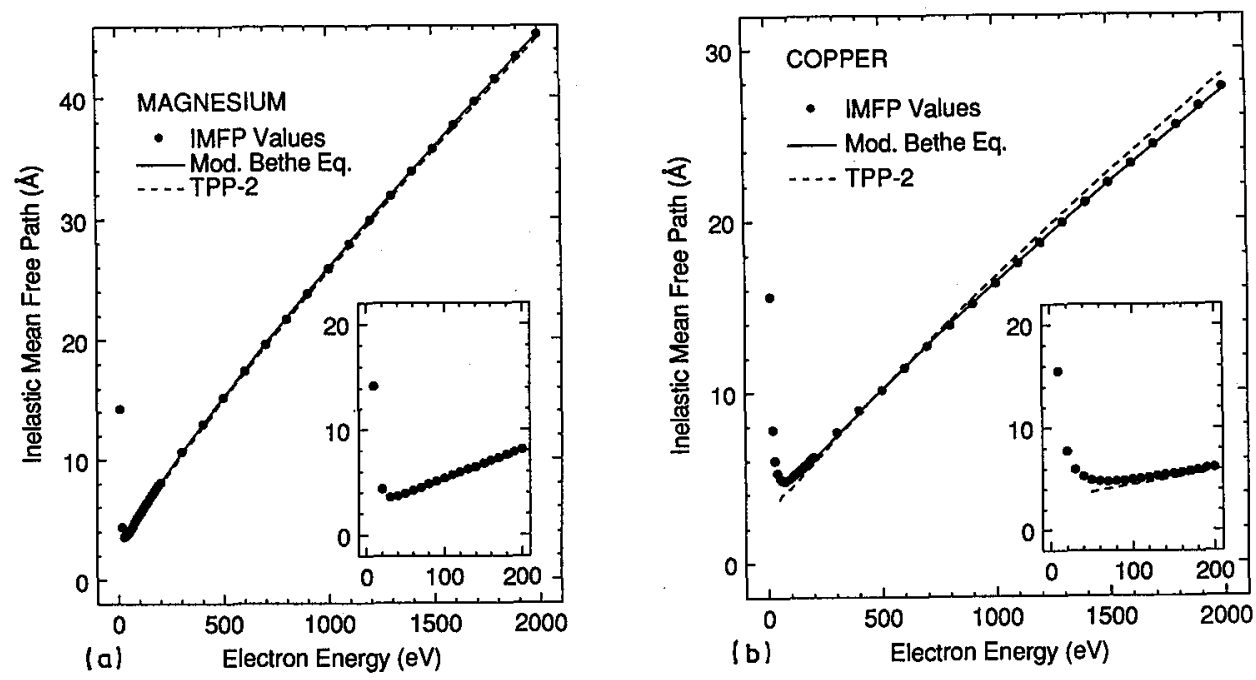

Fig. 1. IMFP values (solid circles) calculated for magnesium (a) and copper (b) as a function of electron energy. IMFP values are shown for 10-40 eV electrons to illustrate trends but these values are not considered reliable (see text). The solid line is a fit to the IMFP values with the modified Bethe equation [Eq. (2)]. The dashed line shows IMFP values calculated from our predictive formula TPP-2 [Eqs. (2) and (3)]. The inset shows the low-energy region on an expanded energy scale. 
Plots of IMFP versus energy are shown in Figs. 1-3 for $\mathrm{Mg}, \mathrm{Cu}, \mathrm{Ru}, \mathrm{Rh}, \mathrm{Ir}$, $\mathrm{GaP}, \mathrm{InP}, \mathrm{LiF}, \mathrm{NaCl}$ and $\mathrm{PbTe}$ as examples of our results. IMFP data are given elsewhere for $\mathrm{C}, \mathrm{Mg}, \mathrm{Al}, \mathrm{Si}$, Ti, V, Cr, Fe, Ni, Y, Zr, Nb, Mo, Pd, Ag, IIf, W, Re, Os, $\mathrm{Pt}, \mathrm{Au}, \mathrm{Bi}, \mathrm{Al}_{2} \mathrm{O}_{3}, \mathrm{GaAs}, \mathrm{InAs}, \mathrm{InSb}, \mathrm{KCl}, \mathrm{PbS}, \mathrm{SiC}, \mathrm{Si}_{3} \mathrm{~N}_{4}, \mathrm{SiO}_{2}$, and $\mathrm{ZnS}$ $[6,14,15]$.

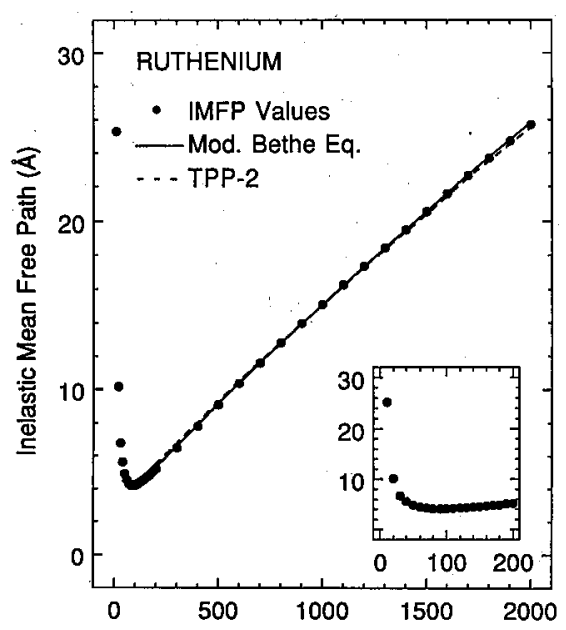

(a) Electron Energy (eV)

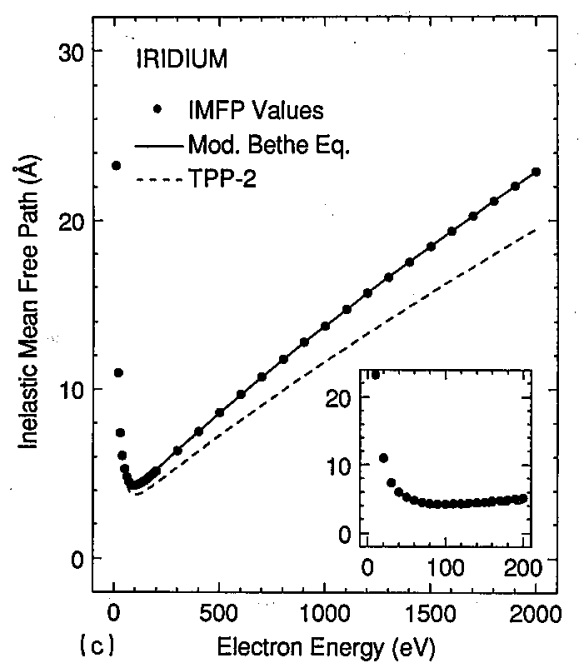

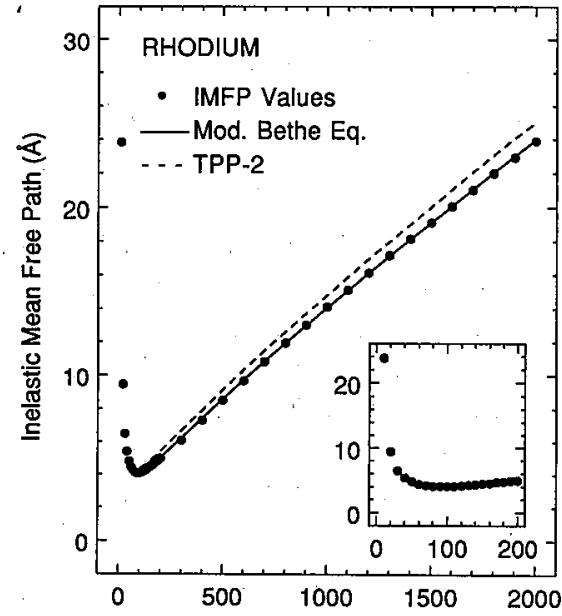

(b) Electron Energy (eV)

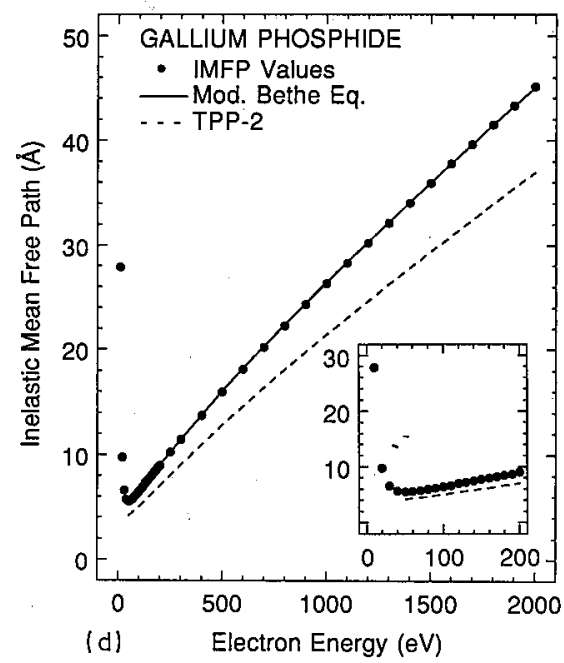

Fig. 2. IMFP results for: ruthenium (a), rhodium (b), iridium (c) and gallium phosphide (d) as a function of electron energy (for other detailes see caption to Fig. 1). 


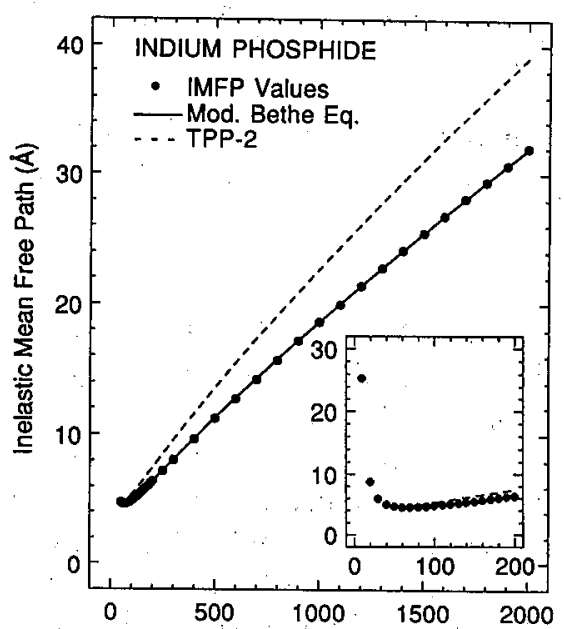

(a) Electron Energy (eV)

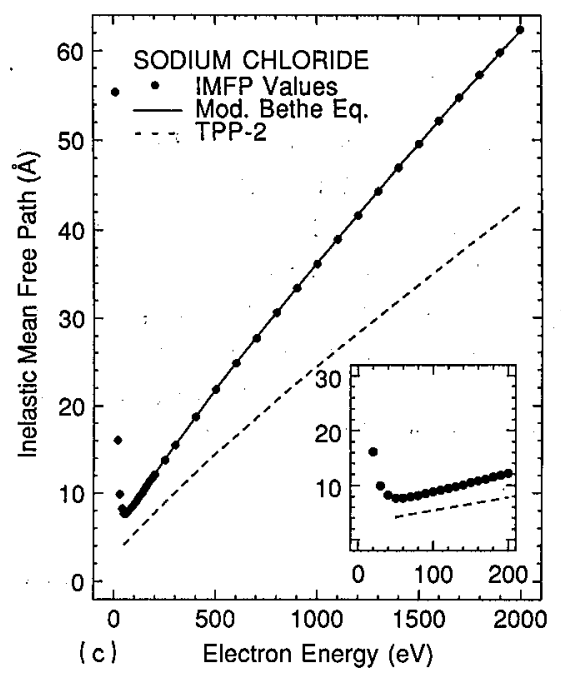

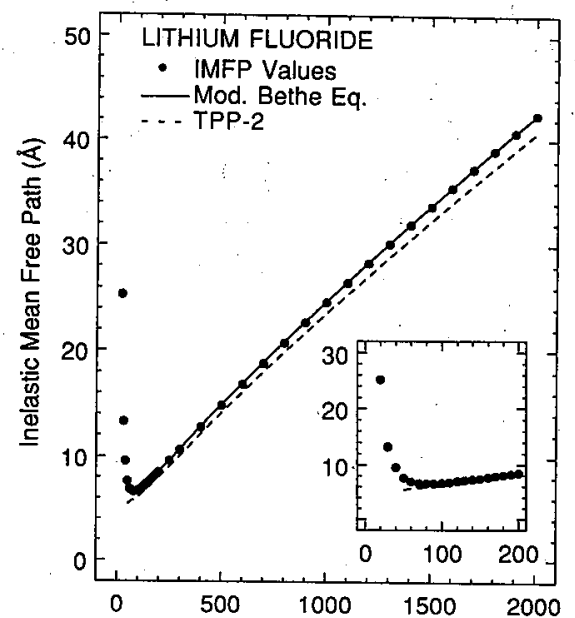

(b) Electron Energy (eV)

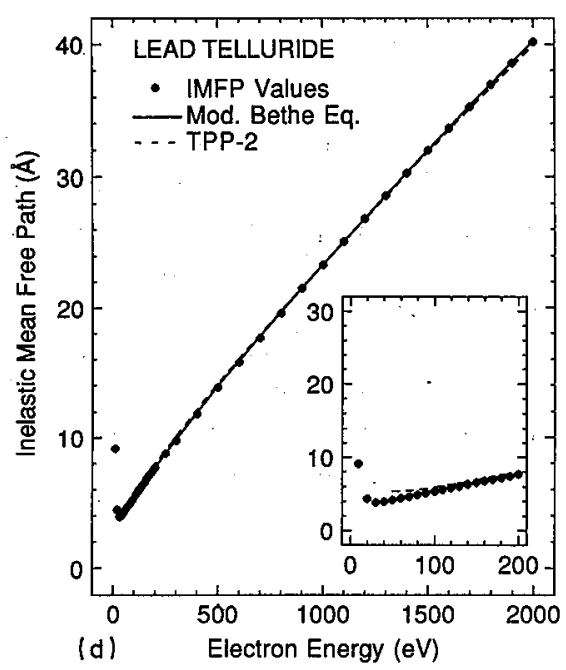

Fig. 3. IMFP results for: indium phosphide (a), lithium fluoride (b), sodium chloride (c) and lead telluride (d) as a function of electron energy (for other detailes see caption to Fig. 1).

The insets in Figs. 1-3 show IMFP values that were calculated from Eq. (14) of Ref. [12] for electron energies between 10 and $40 \mathrm{eV}$; these values are included to illustrate the IMFP trends for energies below the IMFP minimum but these values should be regarded only as rough estimates [6]. The insets also show the qualitative differences in the shapes of the IMFP-energy curves at energies below $200 \mathrm{eV}$ that was discussed earlier [14]. For some of the materials, such as $\mathrm{Mg}, \mathrm{GaP}$, 
$\mathrm{NaCl}$, and $\mathrm{PbTe}$, the IMFP decreases rapidly with increasing energy to a minimum in the range $30-55 \mathrm{eV}$ and then increases; for other materials, such as $\mathrm{Cu}, \mathrm{Ru}, \mathrm{Rh}$, Ir, InP, and LiF, the IMFP decreases more slowly to a broad minimum in the $70-95 \mathrm{eV}$ range.

We have shown earlier $[12,16]$ that the energy dependence of both IMFPs and ALs over the 200-2000 eV energy range can be well described by the Bethe [11] equation for inelastic electron scattering

$$
\lambda=E /\left[E_{\mathrm{p}}^{2} \beta \ln (\gamma E)\right]
$$

where $\lambda$ is the IMFP (in $\AA$ ), $E$ is the electron energy (in eV), $E_{\mathrm{p}}=28.8\left(N_{\mathrm{v}} \rho / M\right)^{1 / 2}$ is the free-electron plasmon energy (in eV), $\rho$ is the bulk density (in $\mathrm{g} \mathrm{cm}^{-3}$ ), $N_{\mathrm{v}}$ is the number of valence electrons per atom or molecule, $M$ is the atomic or molecular weight, and $\beta$ and $\gamma$ are parameters to be determined. The Bethe equation has proven to be useful in the analysis of excitation and ionization cross-sections [18-20], and is expected to be valid if the electron energy is sufficiently high (as will be discussed further below). In the energy range of present interest (50-2000 eV), it is necessary to add two terms to Eq. (1), as proposed by Inokuti [17] and Ashley [21], to account in an empirical way for exchange effects and for other departures from the first Born approximation on which Eq. (1) is based [17]. The modified Bethe equation is:

$$
\lambda=E /\left\{E_{\mathrm{p}}^{2}\left[\beta \ln (\gamma E)-C / E+D / E^{2}\right]\right\}
$$

where $C$ and $D$ are two additional parameters.

Our IMFPs for the elements and compounds have been fitted to Eq. (2) and values of $\beta, \gamma, C$ and $D$ determined for each material [6]. The solid lines in Figs. 1-3 show examples of these fits. We have further analyzed the parameter values for the group of 27 elements to seek relationships between the values of $\beta, \gamma, C$ and $D$ and various material parameters (such as number of valence electrons per atom or molecule, bulk density, and atomic weight); we were guided in this objective by expressions developed previously [21,22] and then examined remaining residuals to find evidence of trends. We obtained the following empirical relations [6]:

$$
\begin{aligned}
& \beta=-0.0216+0.944 /\left(E_{\mathrm{p}}^{2}+E_{\mathrm{g}}^{2}\right)^{1 / 2}+7.39 \times 10^{-4} \rho, \\
& \gamma=0.191 \rho^{-0.50}, \\
& C=1.97-0.91 U, \\
& D=53.4-20.8 U, \\
& U=N_{\mathrm{v}} \rho / M
\end{aligned}
$$

where $E_{\mathrm{g}}$ is the band-gap energy (in eV) for non-conductors. Equations (2) and (3) constitute our formula TTP-2 for predicting IMFPs in other materials.

The dashed lines in Figs. 1-3 show IMFP values obtained from TPP-2. For the group of elements, we found that the average RMS difference between the 
IMFPs calculated from the optical data and those obtained from TPP-2 was about $13 \%$. This level of agreement was considered to be satisfactory given the uncertainties of the optical data used in the IMFP calculation [12] and the empirical nature of TPP-2. For the group of compounds, however, the average RMS difference between the calculated IMFPs and the values from TPP-2 was about $23 \%$, almost double the average difference found for the elements. Furthermore, for most of the compounds, the IMFPs obtained from TPP-2 were systematically lower than those calculated from the optical data (as illustrated by Figs. 2-3 for GaP, LiF, and $\mathrm{NaCl}$ ). These results are discussed further below.

\section{Evaluation of optical data}

Our IMFP calculation is based on values of the electron energy-loss function $\operatorname{Im}[-1 / \varepsilon(\omega)]$ obtained mostly by experiment over typically the $1-10,000 \mathrm{eV}$ range of photon energies. The optical data were evaluated with two useful integral equations or sum rules, the oscillator strength or $f$-sum rule and a limiting form of the Kramers-Kronig integral [23-26]. The $f$-sum is the effective number of electrons per atom or molecule $Z_{\text {eff }}$ contributing to the inelastic scattering:

$$
Z_{\text {eff }}=\left(2 / \pi \hbar^{2} \Omega_{\mathrm{p}}^{2}\right) \int_{0}^{\Delta E_{\max }} \Delta E \operatorname{Im}[-1 / \varepsilon(\Delta E)] \mathrm{d}(\Delta E)
$$

where $\Omega_{\mathrm{p}}=\left(4 \pi n_{a} e^{2} / m\right)^{1 / 2}, n_{a}=N_{a} \rho / M$ is the density of atoms or molecules, $N_{a}$ is Avogadro's number, $\rho$ is the bulk density, $M$ is the atomic or molecular weight, and $\Delta E=\hbar \omega$ is the excitation energy in an inelastic scattering event. When the upper limit in Eq. (4) $\Delta E_{\max }=\infty, Z_{\text {eff }}$ should be equal to $Z$, the total number of electrons per atom or molecule.

The Kramers-Kronig (KK) relations $[23,24]$ can be utilized to calculate $\operatorname{Re}[1 / \varepsilon(\omega)]$ from $\operatorname{Im}[1 / \varepsilon(\omega)]$ if values of the latter quantity are available over a sufficiently wide frequency range. It is convenient to define a quantity $P_{\text {eff }}$ from a simplified form of the KK relations when $\operatorname{Re}[1 / \varepsilon(\omega)]$ is calculated in the limit $\omega \rightarrow 0$ :

$$
P_{\text {eff }}=(2 / \pi) \int_{0}^{\Delta E_{\max }}(1 / \Delta E) \operatorname{Im}[-1 / \varepsilon(\Delta E)] \mathrm{d}(\Delta E)+\operatorname{Re}[1 / \varepsilon(0)] .
$$

For conductors, $\operatorname{Re}[1 / \varepsilon(0)]$ is zero and, in the limit $\Delta E_{\max } \rightarrow \infty$, Eq. (5a) becomes the perfect-screening sum rule that we applied in our analysis of optical data for the elements [12]. For non-conductors, the refractive index $n$ is much greater than the extinction coefficient $k$ at low frequencies and Eq. (5a) becomes:

$$
P_{\text {eff }}=(2 / \pi) \int_{0}^{\Delta E_{\max }}(1 / \Delta E) \operatorname{Im}[-1 / \varepsilon(\Delta E)] \mathrm{d}(\Delta E)+n^{-2}(0)
$$

where $n(0)$ is the limiting value of $n$ as $\omega \rightarrow 0$. In the limit $\Delta E_{\max } \rightarrow \infty, P_{\text {eff }} \rightarrow 1$. Equation (5b) will be termed the KK-sum.

Our analysis of the optical data for the group of elements indicated that the average deviations of the values of $Z_{\mathrm{eft}}$ and $P_{\mathrm{eff}}$ from the expected values about $10 \%$ [12]. These deviations were considered small enough to permit useful IMFP calculations from the optical data and useful analyses of the IMFP trends from material to material. 
For the group of compounds, however, the deviations of $Z_{\text {eff }}$ from $Z$ ranged from $-13 \%$ to $\sim 12 \%$, and the deviations of $P_{\text {eff }}$ from unity ranged from $-40 \%$ to at least $12 \%$ [6], as shown in Table. More significantly, the deviations were negative for most of the compounds, a result which indicates that the optical values of $\operatorname{Im}[-1 / \varepsilon(\omega)]$ were systematically lower than expected; the calculated IMFPs were thus generally larger than if the sum rules had been satisfied.

TABLE

Errors in the $f$-sum rule [Eq. (4)] and the KK-sum rule [Eq. (5b)] for the indicated compounds. Both sums have been evaluated with $\Delta E_{\max }=10,000 \mathrm{eV}$. A minus (plus) indicates that the values of $Z_{\text {eff }}$ and $P_{\text {eff }}$ were less than (greater than) the expected values. We were not able to obtain reliable values of $n(0)$ for. $\mathrm{InSb}, \mathrm{PbTe}$, and $\mathrm{Si}_{3} \mathrm{~N}_{4}$ from Ref. [28]; we show limiting values of the KK-sumerror for these compounds that have been obtained by assuming $n^{-2}(0)$ in Eq. (5b) to be zero. We also show limiting of the $f$-sum errors for the indium and lead compounds due to the neglect of excitations with energies greater than $10^{4} \mathrm{eV}$ in the evaluation of Eq. (4).

\begin{tabular}{l|c|c}
\hline Compound & $\begin{array}{r}\text { Error in } \\
f \text {-sum rule (\%) }\end{array}$ & $\begin{array}{c}\text { Error in } \\
\text { KK-sum (\%) }\end{array}$ \\
\hline $\mathrm{Al}_{2} \mathrm{O}_{3}$ & -6 & -25 \\
$\mathrm{GaAs}$ & -13 & -37 \\
$\mathrm{GaP}$ & -8 & -17 \\
$\mathrm{InAs}$ & $>-5$ & -31 \\
$\mathrm{InP}$ & $>12$ & 3 \\
$\mathrm{InSb}$ & $>-10$ & $>-40$ \\
$\mathrm{KCl}$ & -1 & -25 \\
$\mathrm{LiF}$ & -5 & -30 \\
$\mathrm{NaCl}$ & -5 & -32 \\
$\mathrm{PbS}$ & $>-13$ & -11 \\
$\mathrm{PbTe}$ & $>-12$ & $>12$ \\
$\mathrm{SiC}$ & -2 & -7 \\
$\mathrm{Si}{ }_{3} \mathrm{~N}_{4}$ & -8 & $>-34$ \\
$\mathrm{SiO}$ & -6 & -5 \\
$\mathrm{ZnS}$ & -7 & -19
\end{tabular}

The calculated IMFP at any electron energy is proportional to an integral of $\operatorname{Im}[-1 / \varepsilon(\omega, q)]$ and momentum-transfer terms over the allowed ranges of energy loss and momentum transfer [9]. We use the Lindhard formulation to describe the $q$-dependence of $\operatorname{Im}[-1 / \varepsilon(\omega, q)]$. The main factor determining the magnitude of the computed IMFP is the energy-loss function $\operatorname{Im}[-1 / \varepsilon(\omega)]$. The different depen- 
dences of this function on photon energy (or energy loss in an inelastic scattering event) from material to material lead to the different dependences of the IMFP on electron energy, particularly for energies less than $200 \mathrm{eV}$, as shown in Figs. 1-3.

We show plots of energy-loss functions for $\mathrm{ZnS}, \mathrm{InP}, \mathrm{LiF}$, and $\mathrm{PbTe}$ in Figs. 4 to illustrate the photon energy ranges which have the largest values of $\operatorname{Im}[-1 / \varepsilon(\omega)]$. The differential scattering cross-section with respect to energy loss $\hbar \omega$ is proportional to $\operatorname{Im}[-1 / \varepsilon(\omega)]$, and the regions of energy loss in Figs. 4 which have the largest values of $\operatorname{Im}[-1 / \varepsilon(\omega)]$ will therefore give the greatest contribution to the calculated IMFP. For the four compounds of Figs. 4, the most probable excitation energy is between 10 and $25 \mathrm{eV}$. Excitations of core electrons with binding energies greater than about $100 \mathrm{eV}$ (as shown by the insets of Figs. 4) give very small contributions to the loss functions.
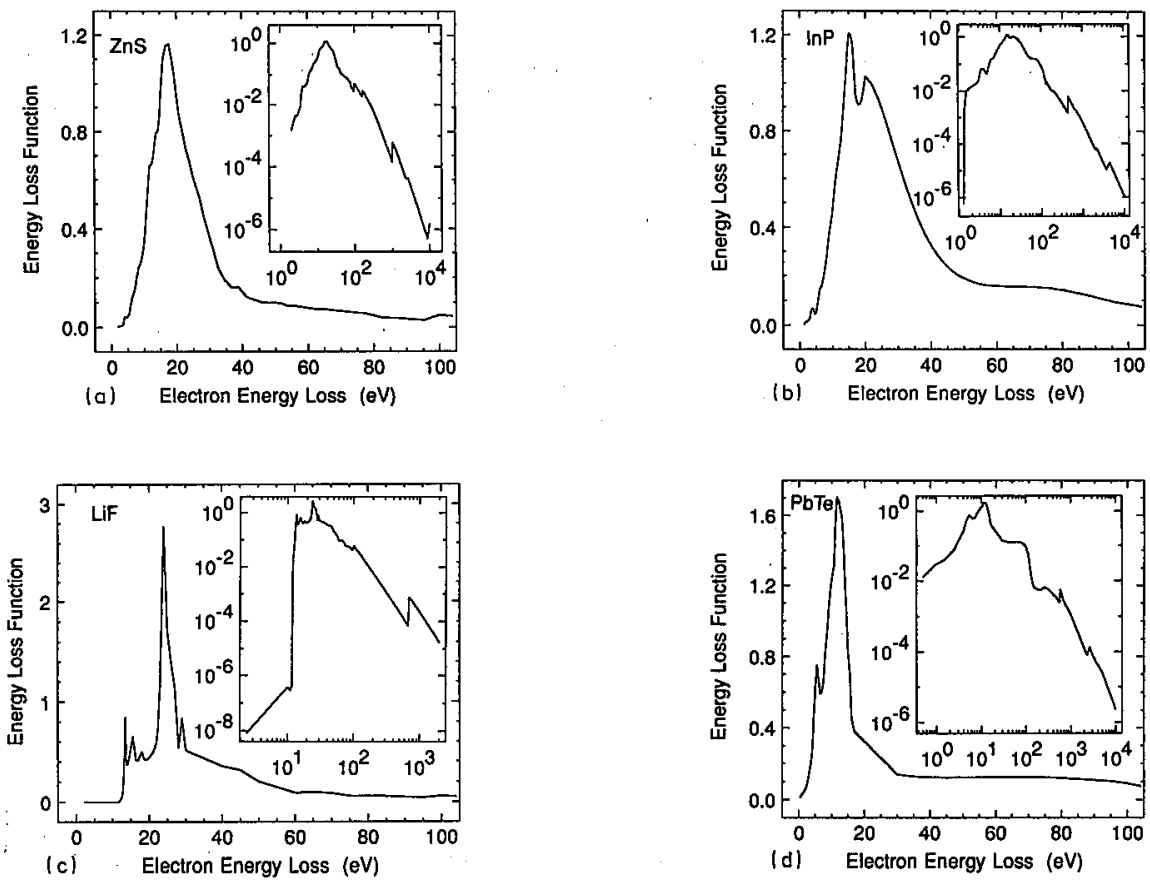

Fig. 4. Plot of the energy-loss function $\operatorname{Im}[-1 / \varepsilon(\omega)]$ for: $\operatorname{ZnS}(\mathrm{a}), \operatorname{InP}(\mathrm{b}), \operatorname{LiF}(\mathrm{c})$ and PbTe (d) as a function of electron energy loss $\hbar \omega$, as calculated from optical data [6]. The main plot shows the $0-100 \mathrm{eV}$ region where the loss function is largest. The inset shows the loss function over a wider energy range (on logarithmic scales).

We show evaluations in Figs. 5 and 6 of the sum rules, Eqs. (4) and (5b), as a function of $\Delta E_{\max }$ for the same four compounds. These evaluations have been performed up to $\Delta E_{\max }=10^{4} \mathrm{eV}$. Since Eq. (4) involves a product of the loss function and excitation energy, the resulting values of $Z_{\text {eff }}$ tend to be dominated for most of the materials considered here by the contributions of core-electron 


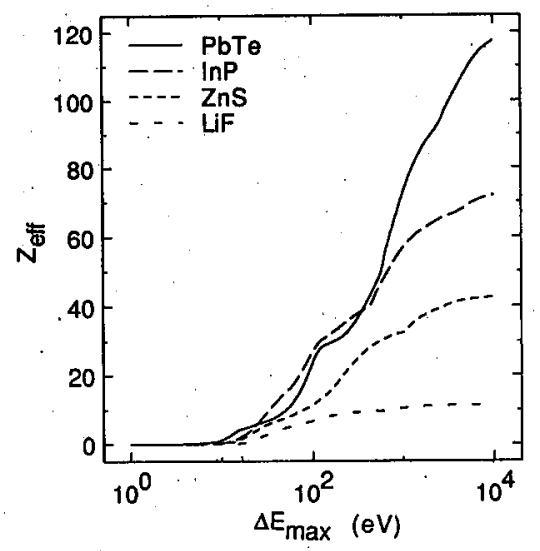

Fig. 5. Plot of $Z_{\text {eff }}$ from Eq. (4) as a function of $\Delta E_{\max }$ for $\mathrm{ZnS}, \mathrm{InP}, \mathrm{LiF}$, and $\mathrm{PbTe}$. The plots of $Z_{\text {eff }}$ should saturate at the values of $Z$ as $\Delta E_{\max } \rightarrow \infty ; Z=12,46,64$, and 134 for $\mathrm{LiF}, \mathrm{ZnS}, \mathrm{InP}$, and $\mathrm{PbTe}$, respectively.

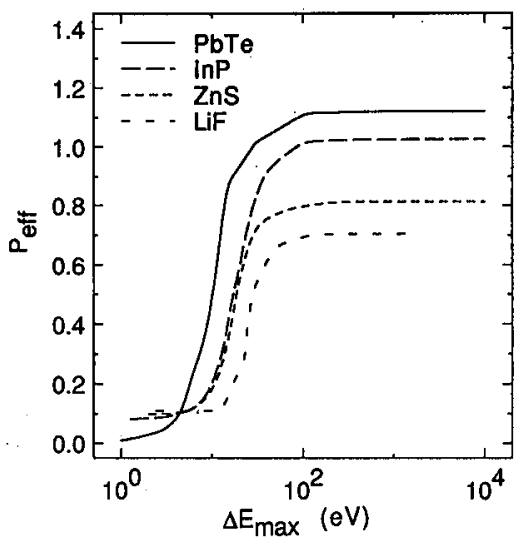

Fig. 6. Plot of $P_{\text {eff }}$ from Eq. (5) as a function of $\Delta E_{\max }$ for $\mathrm{ZnS}, \mathrm{InP}, \mathrm{LiF}$, and PbTe.

excitations. For $\mathrm{LiF}$ and $\mathrm{ZnS}$, the saturation values of $Z_{\text {eff }}$ are 11.4 and 42.6 , respectively; these values are lower than the corresponding values of $Z$ (12 and 46). For InP, the maximum value of $Z_{\text {eff }}$ in Fig. 5 is 71.8 which is greater than the value of $Z$ (64); this discrepancy between $Z_{\text {eff }}$ and $Z$ would be greater if $\Delta E_{\max }$ was increased to include the contributions of the two In $K$-shell electrons with binding energies of $27.9 \mathrm{keV}$ [27]. The maximum value of $Z_{\text {eff }}$ in Fig. 5 for PbTe is 117.6; this value is less than the value of $Z$ (134) but the discrepancy would be reduced by consideration in Eq. (4) of the contributions of the two $K$-shell electrons of $\mathrm{Te}$ and the ten $K$ - and $L$-shell electrons of $\mathrm{Pb}$ which all have binding 
energies greater than $10 \mathrm{keV}$ [27]. We have chosen not to make adjustments to the determinations of $Z_{\text {eff }}$ for the indium and lead compounds associated with excitations greater than $10^{4} \mathrm{eV}$ since the $f$-sum errors are smaller and therefore less significant than the KK-sum errors.

Figure 6 shows plots of $P_{\text {eff }}$ as a function of $\Delta E_{\max }$. The four curves saturate at maximum values of $0.70,0.81,1.03$, and 1.12 for $\mathrm{LiF}, \mathrm{ZnS}, \mathrm{InP}$, and $\mathrm{PbTe}$, respectively (instead of the expected value of unity). The curves reach within $1 \%$ of their maximum values when $\Delta E_{\max }>100 \mathrm{eV}$.

The extent to which measured optical constants for a selected material satisfy sum rules can be a means for gaining confidence in the data and also a useful guide for making adjustments to the data. We have emphasized here the sum rules for $\operatorname{Im}[-1 / \varepsilon(\omega)]$ since these are most relevant to our IMFP calculation, but there are additional sum rules for other optical parameters [23]. We could use such rules together with assessments of possible artifacts due to surface impurities and roughness [28] for data analysis and modification, as has been done with the optical data for $\mathrm{Al}$ [29], but such analyses are beyond the scope of this investigation.

The compounds selected for analysis in Figs. 5 and 6 are examples of groups of compounds which have qualitatively different errors for the $f$-sum and KK-sum rules (Table). $\mathrm{ZnS}$ is one of the compounds (a group that also includes $\mathrm{Al}_{2} \mathrm{O}_{3}$, GaAs, and, tentatively, $\mathrm{InSb}$ and $\mathrm{Si}_{3} \mathrm{~N}_{4}$ that have large negative errors in the KK-sum rule (25-40\%) and smaller negative errors $(6-13 \%)$ in the $f$-sum rule. For these compounds, it is likely that the optical values of $\operatorname{Im}[-1 / \varepsilon(\omega)]$ are less than the correct values over a substantial photon energy range including the range most important for the IMFP calculation, $5-100 \mathrm{eV}$, as illustrated in Fig. 4a for $\mathrm{ZnS}$. $\mathrm{GaP}, \mathrm{PbS}$, and $\mathrm{SiC}$ have intermediate negative errors in the $\mathrm{KK}$-sum rule $(7-17 \%)$ and smaller negative errors in the $f$-sum rule (2-13\%); it is likely that the optical values of $\operatorname{Im}[-1 / \varepsilon(\omega)]$ for these compounds are also underestimated. LiF is one of the compounds (a group including $\operatorname{InAs}, \mathrm{KCl}$, and $\mathrm{NaCl}$ ) that have $25-30 \%$ negative errors in the KK-sum rule and negative errors of $1-5 \%$ in the $f$-sum rule. It is likely that the optical values of $\operatorname{Im}[-1 / \varepsilon(\omega)]$ are correct at relatively high photon energies, including the 5-100 eV range of interest for our IMFP results, as depicted in Fig. 4c for $\mathrm{LiF} . \mathrm{PbTe}$ and $\mathrm{SiO}_{2}$ are the only compounds for which the KK-sum errors are positive and the $f$-sum errors are negative (6-12\%). Here, the optical values of $\operatorname{Im}[-1 / \varepsilon(\omega)]$ may be overestimated at low energies, including the 5-100 eV range (Fig. 4d), and slightly underestimated at higher energies. InP is the only compound that has both a positive $f$-sum error $(12 \%)$ and a positive KK-sum error (3\%). For this compound, the optical values of $\operatorname{Im}[-1 / \varepsilon(\omega)]$ are probably slightly overestimated at most energies.

Finally, we show plots of the quantity $M_{\text {tot }}^{2}$ in Fig. 7 for $\mathrm{ZnS}, \mathrm{InP}, \mathrm{LiF}$, and $\mathrm{PbTe}$. This quantity is the square of the dipole matrix element for all possible inelastic scattering processes and is defined by [17]:

$$
M_{\mathrm{tot}}^{2}=\left(2 R / \pi \hbar^{2} \Omega_{\mathrm{p}}^{2}\right) \int_{0}^{\Delta E_{\max }} \operatorname{Im}[-1 / \varepsilon(\Delta E)] \mathrm{d}(\Delta E)
$$

for $\Delta E_{\max }=\infty$. The term $R$ in Eq. (6) is the Rydberg energy $(13.606 \mathrm{eV})$.

Equation (6) is of interest for two reasons. First, the plots of $M_{\text {tot }}^{2}$ versus 


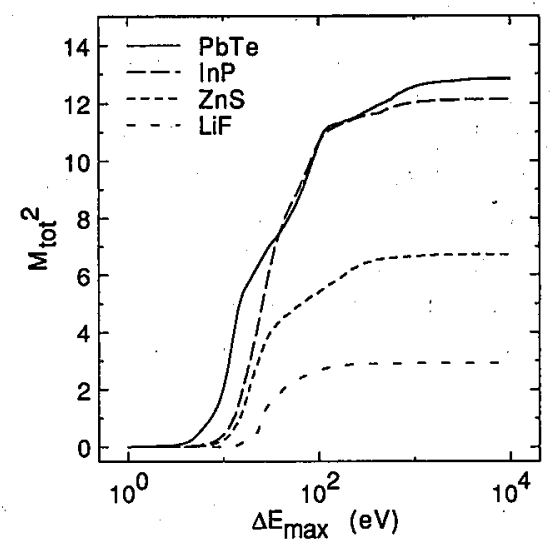

Fig. 7. Plot of $M_{\text {tot }}^{2}$ from Eq. (6) as a function of $\Delta E_{\max }$ for $\mathrm{ZnS}, \mathrm{InP}, \mathrm{LiF}$, and PbTe.

$\Delta E_{\max }$ in Fig. 7 for the four compounds indicate clearly the regions of excitation energy that are most significant in determining the magnitudes of the IMFPs. The plots of Fig. 7 show that the $M_{\text {tot }}^{2}$ values have reached close to their maximum values when $\Delta E_{\max } \geq 300 \mathrm{eV}$. The details of the increase in $M_{\text {tot }}^{2}$ as $\Delta E_{\max }$ increases from 10 to $100 \mathrm{eV}$ depend on the material (that is, the energy-loss function), but subsequent increases due to the excitations of core electrons are relatively small.

The second reason for interest in Eq. (6) is that the value of $M_{\text {tot }}^{2}$ can be used to determine the total inelastic scattering cross-section $\sigma_{\text {tot }}$ from the Bethe equation $[11,17]$ :

$$
\sigma_{\mathrm{tot}}=\left(4 \pi a_{0}^{2} R / E\right) M_{\mathrm{tot}}^{2} \ln \left(4 c_{\mathrm{tot}} E / R\right)
$$

where $a_{0}$ is the Bohr radius $(0.529 \AA)$ and $c_{\text {tot }}$ is a complicated function of the $q$-dependence of $\operatorname{Im}[-1 / \varepsilon(\omega, q)]$. We identify the product $\left(4 c_{\text {tot }} / R\right)$ with our parameter $\gamma$ in Eq. (1). Comparison of Eqs. (1) and (7) yields

$$
\beta_{\mathrm{opt}}=M_{\mathrm{tot}}^{2} / 28.8 N_{\mathrm{v}} \quad\left[\mathrm{eV}^{-1} \AA^{-1}\right]
$$

where $\beta_{\text {opt }}$ is the value of $\beta$ in Eq. (1) determined from the integration of the optical values of $\operatorname{Im}[-1 / \varepsilon(\omega)]$, as indicated by Eqs. (6) and (8).

Figure 8 shows a plot of the values of $\beta$ for our group of elements versus $M_{\text {tot }}^{2} / N_{\mathrm{v}}$, as suggested by Eq. (8). The plot is linear, as expected, but the slope is $0.038=1 / 26.3$, about $10 \%$ greater than expected from Eq. (8). The dashed line in Fig. 9 is a plot of $\beta_{\text {opt }}$ from Eq. (8). The result that $\beta$ exceeds $\beta_{\text {opt }}$ is not surprising since the Bethe equation is not expected to be valid unless the electron energy is sufficiently high [17]. A detailed analysis of the dependence of our calculated IMFPs on energy for $\mathrm{Mg}, \mathrm{Al}, \mathrm{Si}$, Hf and $\mathrm{Bi}$ [6] indicates that the asymptotic value of $\beta$ in Eq. (1) is being approached at $2 \mathrm{keV}$, the maximum energy for our IMFP calculation. It is thus important that our IMFP predictive formula TPP-2 [Eqs. (2) and (3)] would not be extrapolated to higher energies. 


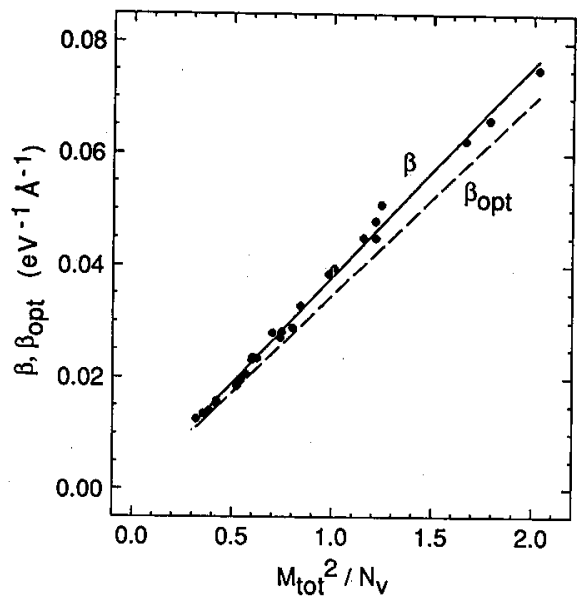

Fig. 8. Plot of values of $\beta$ (solid circles) determined from our fits of Eq. (2) to the IMFPs calculated for our group of elements over the $50-2000 \mathrm{eV}$ range versus $M_{\mathrm{tot}}^{2} / N_{\mathrm{v}}$ where $M_{\text {tot }}$ for each element has been evaluated from $\mathrm{Eq}$. (6) with $\Delta E_{\max }$ generally equal to $10,000 \mathrm{eV}$. The solid line is a least-squares linear fit to the plotted data and the dashed line is a plot of $\beta_{\text {opt }}$ given by Eq. (8).

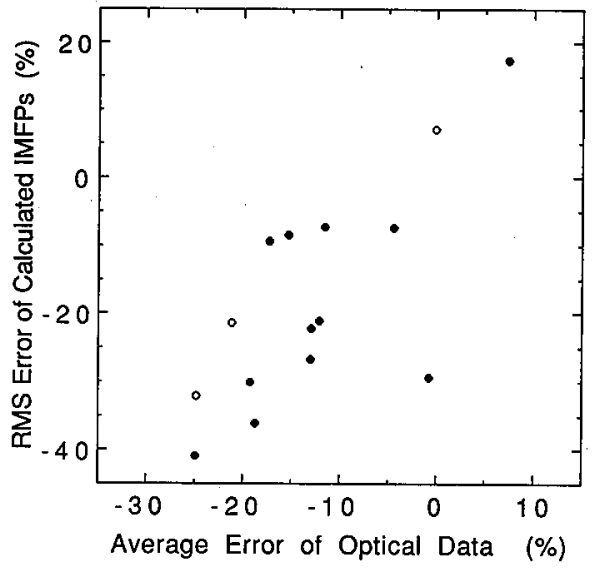

Fig. 9. Plot of the RMS errors in the comparison of IMFPs calculated from optical data for the group of 15 inorganic compounds with the values expected from our IMFP predictive formula TPP-2 [Eqs. (2) and (3)] versus the average of the errors in the $f$-sum and KK-sum rules (Table). Points for $\mathrm{InSb}, \mathrm{PbTe}$, and $\mathrm{Si}_{3} \mathrm{~N}_{4}$ are shown as open circles; for these compounds the KK-sum errors are known only as lower limits and thus the negative average optical errors are overestimated. Points for the other compounds are shown as solid circles. 


\section{Discussion}

Our analysis of the optical values of $\operatorname{Im}[-1 / \varepsilon(\omega)]$ for the group of 15 inorganic compounds has shown significant shortcomings of these data, as indicated by the sum-rule errors listed in Table. We have found that the discrepancies between the IMFPs calculated from the optical data and the values predicted by TPP-2 [Eqs. (2) and (3)] are correlated with the sum-rule errors. Figure 9 shows the RMS errors in the comparison of IMPPs from the optical data with TPP-2 values plotted versus the average of the errors in the $f$-sum and KK-sum rules (Table). Although these sum rules emphasize $\operatorname{Im}[-1 / \varepsilon(\omega)]$ values in energy regions that are higher and lower, respectively, than the region most important for the IMFP calculation, we consider the average error for these two sum rules to be a physically reasonable (but approximate) indication of the likely accuracy of the optical values of $\operatorname{Im}[-1 / \varepsilon(\omega)]$. Although the $f$-sum rule integration is dominated by the contributions of core electrons for elements of medium and high atomic number (as indicated in Fig. 5), the $f$-sum errors are generally much smaller than the KK-sum errors (Table). As a result, the average errors of the optical data are mainly determined by errors of the KK-sum rule.

The approximately linear relationship in Fig. 9 is a strong indication that there are systematic errors in the IMFP values for our compounds due to inadequacies of the optical data. While we could use information from the sum rules for $\operatorname{Im}[-1 / \varepsilon(\omega)]$ and other optical functions [23] to adjust the optical data, we have chosen not to do this, partly because of the amount of work involved and partly because of the possibility of arbitrary choices that might have to be made. Instead, we believe that IMFPs obtained from TPP 2 provide reasonable estimates for these materials. We note that the RMS error in the use of TPP-2 for calculating IMFPs of the group of 27 elements was $13 \%$. The scatter of points in Fig. 9 about the correlation line is consistent with the RMS error in the use of TPP-2 for the group of elements. It is therefore expected that there will be a similar uncertainty in the use of TPP-2 for predicting relative IMFPs of inorganic compounds. Other factors which may influence the absolute accuracy of IMFPs from TPP-2 are discussed elsewhere [6].

Calculations of IMFPs from TPP-2 require an appropriate choice of parameters. We have examined the extent to which the calculated IMFPs change as individual parameters in TPP-2 are varied in physically reasonable ranges [6]. Figures 10-12 show IMFP versus energy curves for copper and the effects of varying in turn the bulk density $\rho$, the number of valence electrons per atom $N_{\mathrm{v}}$, and the free-electron plasmon energy $E_{\mathrm{p}}$. Figure 10 shows that increases of $\rho$ lead to a systematic reduction in the IMFPs calculated from TPP-2 and to some changes in shapes of the IMFP-energy curves for energies less than $200 \mathrm{eV}$. An increase in the value of $N_{\mathrm{v}}$ from 1 to 11 causes a decrease in the computed IMFPs, as indicated in Fig. 11, and also to shape changes in the IMFP-energy curves for $E<200 \mathrm{eV}$. A change of $N_{\mathrm{v}}$ in the range 3 to 15 , however, leads to IMFP changes of less than 20\%; similar calculations for $\mathrm{Au}$ and $\mathrm{Al}$ showed IMFP changes of less than $75 \%$ and $25 \%$, respectively [6]. Finally, an increase of $E_{\mathrm{p}}$ in the range $5-30 \mathrm{eV}$ produces an IMFP decrease as shown in Fig. 12; further increase of $E_{\mathrm{p}}$ leads to 


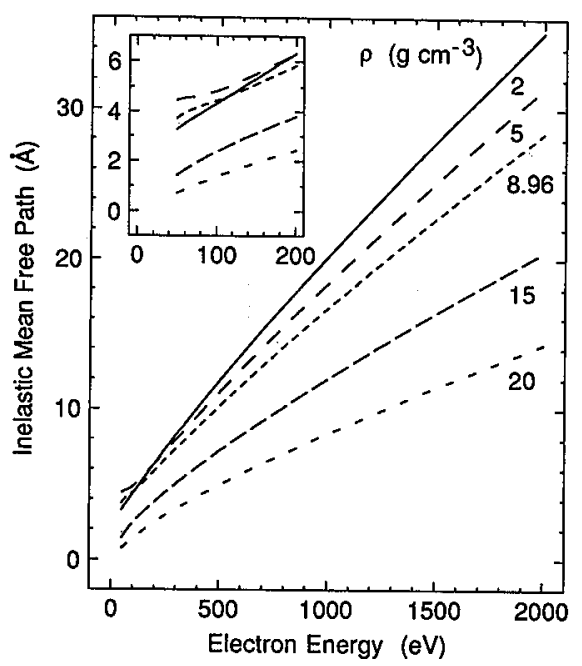

Fig. 10. Plots of IMFP versus energy for Cu from TPP-2 (curve with $\rho=8.96 \mathrm{~g} \mathrm{~cm}^{-3}$ ) and of evaluations of TPP-2 [Eqs. (2) and (3)] with Cu parameters except that the value of the bulk density $\rho$ was varied as shown. The inset shows IMFP values for the $50-200 \mathrm{eV}$ range on an expanded scale.

a slight IMFP increase. There are also substantial changes in shape of the curves for $E<200 \mathrm{eV}$. The results in Figs. 10-12 and the similar calculations for $\mathrm{Al}$ and $\mathrm{Au}$ indicate that the broad minima in the IMFP-energy curves (e.g., as for $\mathrm{Cu}$ in Fig. 1b) are associated with generally higher values of $\rho, N_{\mathrm{v}}$, and $E_{\mathrm{p}}$ than those which give narrower minima (e.g., as for $\mathrm{Mg}$ in Fig. 1).

We now consider whether a simpler or more accurate expression than TPP-2 might be derived for the prediction of IMFPs. The magnitude of an IMFP calculated for a material from TPP-2 at some energy greater than $200 \mathrm{eV}$ is determined largely by the product $\beta E_{\mathrm{p}}^{2}$ which is contained in the leading term of Eq. (2). Since $\beta \approx \beta_{\text {opt }}$, this product $\beta E_{\mathrm{p}}^{2}$ can also be expressed as [12]:

$$
\beta E_{\mathrm{p}}^{2} \approx 28.8 \rho M_{\text {tot }}^{2} / M \quad\left[\mathrm{eV} \AA^{-1}\right]
$$

with $M_{\text {tot }}^{2}$ defined by Eq. (6). The plots of $M_{\text {tot }}^{2}$ versus $\Delta E_{\max }$ in Fig. 7 illustrate the importance of electronic excitations in the $5-100 \mathrm{eV}$ range in determining the value of $M_{\text {tot }}^{2}$ and thus of $\beta E_{\mathrm{p}}^{2}$.

The two sum rules [Eqs. (4) and (5b)] used for evaluation of the optical data involve integrals of frequency moments of the loss function. While these integrals have well-defined values, there is not, unfortunately, a well-defined value for the integral of the loss function itself. That is, the value of $M_{\text {tot }}^{2}$ from the integral of Eq. (6) cannot be simply expressed in terms of other physical properties that depend on electronic excitations. Since it is not possible to provide a simple analytic expression for $M_{\mathrm{tot}}^{2}$, it is similarly not possible for us to give an expression for $\beta E_{\mathrm{p}}^{2}$ 

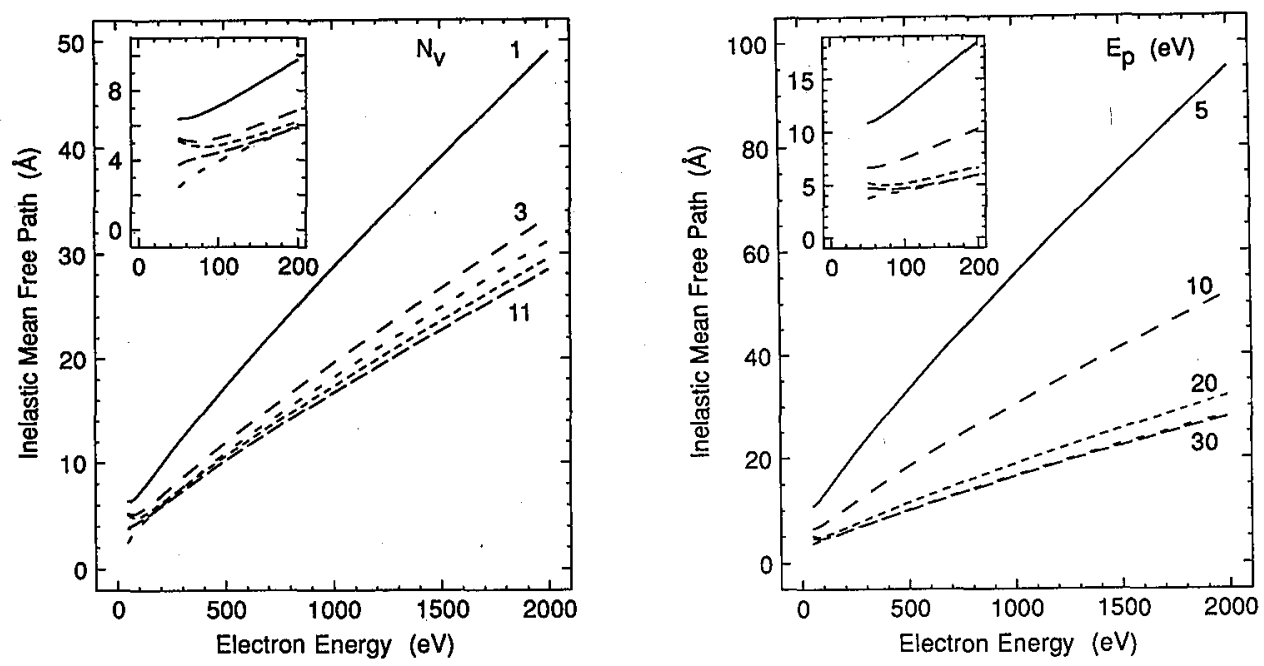

Fig. 11. Plots of IMFP versus energy for Cu from TPP-2 (curve with $N_{\mathrm{v}}=11$ ) and of evaluations of TPP-2 with $\mathrm{Cu}$ parameters except that the number of electrons per atom $N_{\mathrm{v}}$ was varied as shown. The curve adjacent to the $N_{\mathrm{v}}=3$ curve is for $N_{\mathrm{v}}=5$ while the curve adjacent to the bottom $N_{\mathrm{v}}=11$ curve is for $N_{\mathrm{v}}=15$.

Fig. 12. Plots of IMFP versus energy for $\mathrm{Cu}$ from TPP-2 (curve adjacent to the bottom curve, with $E_{\mathrm{p}}=35.9 \mathrm{eV}$ ) and of evaluations of TPP-2 with Cu parameters except that the free-electron plasmon energy was varied as shown.

based on general physical principles.

The other two terms in Eq. (2) account for deviations from the Bethe equation that are important in the 50-200 eV energy range. These correction terms are physically plausible $[17,21]$ but future calculations might give improvements. It is also possible that future work could give a better physical representation than Eq. (3) for the dependence of $\beta, \gamma, C$ and $D$ on material parameters. Until future guidance is available, we consider TPP-2 to be a useful guide for predicting IMPFs. Nevertheless, since our Eq. (3) has been obtained empirically, it is not reasonable to expect that these expressions can represent in detail the variations in inelastic-scattering properties that occur from material to material, particularly for energies less than $200 \mathrm{eV}$.

\section{Summary}

We have calculated IMFPs of 50-2000 eV electrons in 27 elemental solids and 15 inorganic compounds. Substantial variations in the shapes of the IMFP-energy curves from material to material have been found for energies less than $200 \mathrm{eV}$. These variations are associated with detailed differences in the energy-loss functions amongst the materials. 
We have developed a general equation for predicting IMFPs based on the IMFP values for our group of elements. This equation, designated TPP-2, provides a convenient means for estimating IMFPs in other materials and for expressing the IMFP dependence on electron energy. TPP-2 should not, however, be used for electron energies and for material parameters outside the ranges for which it has been developed and tested [6]. The TPP-2 formula represented the IMFPs calculated for our elements with an average RMS uncertainty of about $13 \%$.

We have used two powerful sum rules to evaluate the optical values of $\operatorname{Im}[-1 / \varepsilon(\omega)]$ used in our IMFP calculation. These sum rules were satisfied within an RMS uncertainty of about $10 \%$ for the group of elements but the uncertainties were much larger for the group of inorganic compounds. We found that differences between IMFPs calculated for the compounds from the optical data and values predicted by TPP-2 correlated with average errors of the optical data as revealed by the sum rules. We therefore believe that IMFPs calculated from TPP-2 are more reliable than those computed from the deficient optical data. Our formula TPP-2 is robust and appears to be a useful means for estimating IMFP values and for determining the IMFP dependences on material and energy.

\section{References}

[1] C.J. Powell, J. Electron Spectrosc. Relat. Phenom. 47, 197 (1988).

[2] A. Jabłoński, H. Ebel, Surf. Interface Anal. 11, 627 (1988).

[3] W.H. Gries, W. Werner, Surf. Interface Anal. 16, 149 (1990).

[4] A. Jabłoński, S. Tougaard, J. Vac. Sci. Technol. A 8, 106 (1990).

[5] C.J. Powell, M.P. Seah, J. Vac. Sci. Technol. A 8, 735 (1990).

[6] S. Tanuma, C.J. Powell, D.R. Penn (to be published).

[7] W.S.M. Werner, W.H. Gries, H. Störi, J. Vac. Sci. Technol. A 9, 21 (1991).

[8] Z.-J. Ding, R. Shimizu (to be published).

[9] D.R. Penn, Phys. Rev. B 35, 482 (1987).

[10] J. Lindhard, M. Scharff, Kong. Danske Vidensk. Selk. Matt.-Fys. Medd. 27, No. 15 (1953); J. Lindhard, M. Schaiff, H.E. Schiott, Kong. Danske Vidensk. Selk. Matt.-Fys. Medd. 33, No. 14 (1963); D. Pines, Elementary Excitations in Solids W.A. Benjamin, New York 1963, p. 144.

[11] H.H. Betle, Ann. Phys. (East Germany) 5, 325 (1930).

[12] S. Tanuma, C.J. Powell, D.R. Pemi, Surf. Inlerface Anal. 11, 577 (1988).

[13] B.I. Henke, P. Lee, T.J. Tanaka, R.L. Shimabukuro, B.K. Fujikawa, At. Data Nucl. Data Tables (USA) 27, 1 (1982); E.B. Saloman, J.H. Hubbell, J.H. Scofield, At. Data Nucl. Data Tables (USA) 38, 1 (1988).

[14] S. Tanuma, C.J. Powell, D.R. Penn, J. Vac. Sci. Technol. A 8, 2213 (1990).

[15] S. Tanuma, C.J. Powell, D.R. Penn, J. Electron Spectrosc. Relat. Phenom. 52, 285 (1990).

[16] C.J. Powell, Surf. Interface Anal. 7, 256 (1985); Surf. Interface Anal. 10, 349 (1987).

[17] M. Inokuti, Rev. Mod. Phys. 43, 297 (1971).

[18] F.J. de Heer, M. Inokuti, in: Electron Impact Ionization, Eds. T.D. Märk, G.H. Dunn, Springer-Verlag, New York 1985, p. 232. 
[19] C.J. Powell, in: Electron Impact Ionization, Eds. T.D. Märk, G.H. Dunn, Springer-Verlag, New York 1985, p. 198.

[20] C.J. Powell, in: Microbeam Analysis-1990, Eds. J.R. Michael, P. Ingram, San Francisco Press, San Francisco 1990, p. 13.

[21] J.C. Ashley, J. Electron Spectrosc. Relat. Phenom. 46, 199 (1988).

[22] J. Szajman, J. Liesegang, J.G. Jenkin, R.C.G. Leckey, J. Electron Spectrosc. Relat. Phenom. 23, 97 (1981); J.C. Ashley, J. Electron Spectrosc. Relat. Phenom. 28, 177 (1982).

[23] D.Y. Smith, in: Handbook of Optical Constants of Solids, Ed. E.D. Palik Academic Press, New York 1985, p. 35.

[24] J. Daniels, C. v. Festenberg, H. Raether, K. Zeppenfeld, Springer Tracts in Modern Physics 54, 77 (1970).

[25] D. Pines, P. Nozieres, The Theory of Quantum Liquids, W. Benjamin, New York, 1966, Vol. 1, p. 210.

[26] G.D. Mahan, Many-Particle Physics, Plenum, New York 1981, p. 460.

[27] J.A. Bearden, A.F. Burr, Rev. Mod. Phys. 39, 125 (1967).

[28] E.D. Palik, in: Handbook of Optical Constants of Solids, Ed. E.D. Palik, Acadenic Press, New York 1985, p. 3.

[29] E. Shiles, T. Sasaki, M. Inokuti, D.Y. Smith, Phys. Rev. B 22, 1612 (1980). 